\title{
Thèse remarquée
}

\author{
Développement de modèles dosimétriques en radiothérapie externe par méthodes de \\ Monte Carlo. Études des effets liés à la présence d'implants artificiels. Edwige \\ Buffard. Thèse de doctorat de l'université de Franche-Comté, soutenue le 23 juin 2006 \\ à Montbéliard, Laboratoire d'accueil: IRMA/CREST/FEMTO-ST, UMR 6174 \\ CNRS, direction de thèse : L. Makovicka, encadrement : R. Gschwind.
}

En radiothérapie externe, l'élaboration d'un plan de traitement est menée selon deux objectifs : optimiser l'irradiation du volume cible et épargner au maximum les organes à risque avoisinants. Dans le cas d'un patient porteur d'implants métalliques, qui se trouvent à proximité de la tumeur, ces deux objectifs sont difficiles à atteindre. L'allongement de l'espérance de vie, à l'origine de l'augmentation des implantations de prothèses artificielles, cumulée à une forte incidence du cancer de la prostate renforce la problématique du traitement par radiothérapie externe des patients porteurs de prothèses de hanche. À l'heure actuelle, la difficulté d'établir un plan de traitement efficace pour ces patients repose sur la capacité des systèmes de planification de traitement (TPS) à fournir un calcul précis de la dose en présence d'hétérogénéités de densités élevées. En réponse aux faiblesses des algorithmes de calcul implémentés dans les TPS, les méthodes de Monte Carlo apparaissent comme la technique la plus performante pour déterminer l'impact des prothèses de hanche sur la dose.

L'objectif de ce travail de thèse a donc consisté, d'une part à calculer les modifications de dose produites en présence de prothèses de hanche au cours d'une irradiation du pelvis au moyen du code Monte Carlo OMEGA / BEAMnrc, et d'autre part à proposer une évaluation des systèmes de planification de traitement dans ces cas limites. Une première étude a porté sur la détermination des doses au contact des implants métalliques lorsque le traitement du patient est mené en utilisant un faisceau d'irradiation qui traverse la prothèse. Une attention particulière a notamment été donnée à l'estimation de l'impact des différents revêtements ou ciments, utilisés pour la fixation des prothèses, sur la dose à l'interface tissu-métal. Afin d'optimiser la détermination des distributions de dose, la conception de fantômes anthropomorphiques a été indispensable. Des géométries réalistes ont ainsi été élaborées au moyen du module CTCreate à partir d'images CT de patients. En parallèle, un programme EgsphantOpener a été spécifiquement développé dans le cadre de la thèse afin de gérer la présence des artefacts sur les images CT des patients porteurs d'implants artificiels. Enfin une évaluation de la capacité des quelques TPS à tenir compte de la présence de prothèses de hanche a été proposée. Les simulations Monte Carlo ont servi à établir les performances des formalismes de calcul à estimer la dose dans ces cas limites. 